\title{
33
}

\section{Reliability Considerations of Beams Subjected to Random Moving Loads}

\author{
H. S. Zibdeh ${ }^{a, *}$ and R. Rackwitz ${ }^{b}$ \\ ${ }^{a}$ Jordan University of Science and Technology \\ ${ }^{b}$ Technical University of Munich
}

\begin{abstract}
The reliability of elastic beams under a Poissonian stream of moving random loads is still a subject of intensive research. In particular, the assumption of Gaussianity of the responses is highly questionable for short beams and/or low arrival intensities. As a consequence reliability predictions with respect to extreme value or fatigue failure are inaccurate. In the paper analytical results for the response moments up to fourth order will be derived for different types of motion of the stream of moving loads. The results are then used in studies for extreme and fatigue failure employing Winterstein's expansion of non-Gaussian processes in terms of higher moments. The effect of important parameters, i.e., arrival intensity, velocity, damping ratio is investigated
\end{abstract}

\section{Introduction}

The reliability of a simply supported linear elastic beam subjected to a Poissonian stream of moving mutually independent and identically distributed loads still attracts the attention of many investigators because various aspects are still not fully clarified. In particular the assumption of Gaussianity of the response is highly questionable especially for short beam and/or low arrival rates. Already Tung [1-3] studied the response of highway bridges to random loads moving with the same constant speed. Based on numerical procedures, he obtained the density function of the response and its excursion rate, and he estimated the fatigue life of highway bridges. Sieniawska and Sniady [4] studied the dynamic response of a finite beam to the passage of train of concentrated random forces moving with the same constant speed. They obtained the excursion rate for a given threshold by finding some form for the joint density function of deflection and speed of the response. They also estimated the life of the structure by finding the joint probability density function of the displacement, velocity, and acceleration of the vibrating beam [5]. Fryba [6] estimated the fatigue life of railway bridges where he assumed that the loading is either due to the movement of a random force along the beam or due to an infinite strip of moving continuous random load. Based on the results presented in [7], this paper investigates the excursion rates and fatigue lives of the beam using an analytical model of Winterstein [10]. This model utilizes the higher order moments of the response quantities to account for the non-Gaussian characteristics of the response.

\footnotetext{
${ }^{*}$ Visiting the Technical University of Munich. Research fellow of the Alexander von Humboldt Foundation
} 


\section{Theoretical Formulation}

A simply supported beam, originally at rest, is loaded by a stream of loads moving in the same direction all with the same type of motion as shown in Fig. 1. The loads arrive at the left end of the beam at random times $t_{i}$ which constitute a stationary random process of filtered Poissonian type. This problem is described by the following differential equation

$E I \frac{\partial^{4} v(x, t)}{\partial x^{4}}-N \frac{\partial^{2} v(x, t)}{\partial x^{2}}+M \frac{\partial^{2} v(x, t)}{\partial t^{2}}+2 M \omega_{b} \frac{\partial v(x, t)}{\partial t}=\sum_{i=1}^{N(t)} P_{i} \delta\left(x-f\left(t-t_{i}\right)\right)$

with appropriate boundary and initial conditions where $E I, N, M, \omega_{b}, l$, and $v(x, t)$ denote, respectively, the flexural rigidity of the beam, the axial force applied at the end of the beam, the mass per unit length, the circular frequency of damping, the span length, and the vertical deflection of the beam at point $x$ and time $t$.

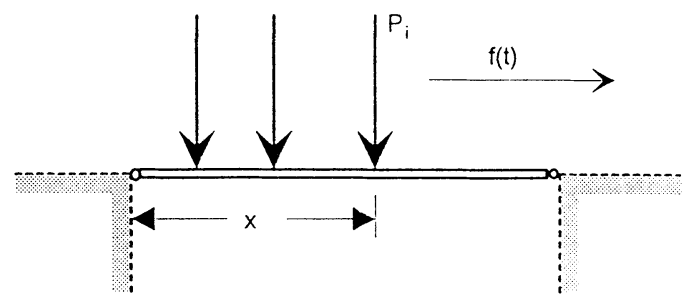

Figure 1. Beam Model

The right hand side of Eq. (1) represents the loading process where $\delta($.$) denotes$ the Dirac delta function, $f(t)$ denotes a function describing the motion of the load at time $t, P_{i}$ are the force amplitudes which are independent, identically distributed random variables independent of their times of arrival $t_{i}$, and $N(t)$ is a Poisson counting process with parameter $\lambda$. In modal form, the vertical deflection of the beam can be written as

$v(x, t)=\sum_{m=1}^{\infty} W_{m}(x) Y_{m}(t)$

where $W_{m}(x)$ are the normal modes of free vibration, and $Y_{m}(t)$ are the generalized deflections or the modal responses. For a simply supported beam there is $W_{m}(x)=$ $\sin \left(\frac{m \pi x}{l}\right)$. Carrying out the familiar operations, the differential equation of the $\mathrm{m}$ th mode of the generalized deflection or the modal response is written as

$\ddot{Y}_{m}+2 \xi \omega_{m} Y_{m}+\omega_{m}^{2} Y_{m}=\frac{2}{M l} \sum_{i=1}^{N(t)} P_{i} \sin \left(\frac{m \pi}{l} f\left(t-t_{i}\right)\right)$

The natural frequency of the undamped beam $\omega_{m}$ is defined as $\omega_{m}^{2}=\omega_{1,0}^{2} m^{2}\left(m^{2} \pm \psi\right)$ with $\omega_{1,0}^{2}=\frac{E I}{M}\left(\frac{\pi}{l}\right)^{4}, \psi=\frac{N}{N_{c r}}$ and $N_{c r}=\frac{\pi^{2} E I}{l^{2}} . \omega_{1,0}$ is the first natural frequency of the beam without axial force, $\stackrel{N}{c r}_{c r}$ is the Euler buckling force, and $f$ is the axial force ratio. 
The coefficient of damping $\xi$ is defined as $\xi=\omega_{b} / \omega_{1}$. Considering the physical nature of the right hand side of Eq. (3) and using the principle of superposition, the solution for the modal response of Eq. (3) is written as [8]

$Y_{m}(t)=\sum_{t-T \leq t_{i} \leq t} P_{i} H_{m}^{I}\left(t, t_{i}, T\right)+\sum_{0 \leq t_{i} \leq t-T} P_{i} H_{m}^{I I}\left(t, t_{i}, T\right)$

where $H_{m}^{I}\left(t, t_{i}, T\right)$ and $H_{m}^{I I}\left(t, t_{i}, T\right)$ are the $\mathrm{m}$ th modal response at time $t$ to a single pulse having the shape $\sin \left(\frac{m \pi}{l} f\left(\tau-t_{i}\right)\right)$ and duration $T$. These functions give the effect of the pulse when it is on the beam, i.e. for $t-t_{i} \leq T$, and when it leaves the beam, i.e. for $t-t_{i}>T$, respectively. These functions are

$H_{m}^{I}\left(t, t_{i}, T\right)=\frac{2}{M l} \int_{t_{i}}^{t} h_{m}(t-\tau) \sin \left(\frac{m \pi}{l} f\left(\tau-t_{i}\right)\right) d \tau$

$H_{m}^{I I}\left(t, t_{i}, T\right)=\frac{2}{M l} \int_{t_{i}}^{T+t_{i}} h_{m}(t-\tau) \sin \left(\frac{m \pi}{l} f\left(\tau-t_{i}\right)\right) d \tau$

where

$h_{m}(t)=\left\{\begin{array}{cc}\frac{1}{\omega_{m}^{\prime}} e^{-\omega_{b} t} \sin \omega \prime_{m} t & t \geq 0 \\ 0 & t<0\end{array}\right.$

and $\omega_{m}^{\prime 2}=\omega_{m}^{2}-\omega_{b}^{2}$.Assuming a quadratic form for the motion of the force along the beam, yields

$f(t)=x_{0}+c t+a \frac{t^{2}}{2}$

where $x_{0}$ is the point of application of the force, $c$ is the initial speed, and $a$ is the constant acceleration. The relation $f(t)=c t$ describes motion with uniform speed. Substituting Eq. (7) into Eqs.(5) and (6) and into Eq. (3) yields the deflection $v(x, t)$ as

$v(x, t)=\sum_{t-T \leq t_{i} \leq t} P_{i} \sum_{m=1}^{\infty} \sin \left(\frac{m \pi x}{l}\right) H_{m}^{I}\left(t, t_{i}, T\right)+\sum_{0 \leq t_{i} \leq t-T} P_{i} \sum_{m=1}^{\infty} \sin \left(\frac{m \pi x}{l}\right) H_{m}^{I I}\left(t, t_{i}, T\right)$

The first term of Eq. (9) gives the total response of the beam to all forces that are on the beam at time $t$. The second term gives the total response of all forces that have left the beam up to timet. The integrations required in Eqs. (5) and (6) are analytic. The final expression for the case of the time varying velocity, for example, is a function of the error function of complex arguments [7,8]. Similarly, the velocity and acceleration of the modal response can be obtained

$$
\begin{aligned}
& \dot{v}(x, t)=\sum_{t-T \leq t_{i} \leq t} P_{i} \sum_{m=1}^{\infty} \sin \left(\frac{m \pi x}{l}\right) \dot{H}_{m}^{I}\left(t, t_{i}, T\right)+\sum_{0 \leq t_{i} \leq t-T} P_{i} \sum_{m=1}^{\infty} \sin \left(\frac{m \pi x}{l}\right) \dot{H}_{m}^{I I}\left(t, t_{i}, T\right) \\
& \ddot{v}(x, t)=\sum_{t-T \leq t_{i} \leq t} P_{i} \sum_{m=1}^{\infty} \sin \left(\frac{m \pi x}{l}\right) \ddot{H}_{m}^{I}\left(t, t_{i}, T\right)+\sum_{0 \leq t_{i} \leq t-T} P_{i} \sum_{m=1}^{\infty} \sin \left(\frac{m \pi x}{l}\right) \ddot{H}_{m}^{I I}\left(t, t_{i}, T\right)
\end{aligned}
$$


The semi-invariants of the distributions of the response and its derivatives can be determined from [9]

$\eta_{n}(x, t)=\lambda E\left[P_{i}^{n}\right]\left[\int_{0}^{T}\left[\sum_{m=1}^{\infty} \sin \left(\frac{m \pi x}{l}\right) H_{m}^{I}\left(s_{i}, T\right)\right]^{n} d s_{i}+\int_{T}^{t}\left[\sum_{m=1}^{\infty} \sin \left(\frac{m \pi x}{l}\right) H_{m}^{I I}\left(s_{i}, T\right)\right]^{n} d s_{i}\right]$
$\dot{\eta}_{n}(x, t)=\lambda E\left[P_{i}^{n}\right]\left[\int_{0}^{T}\left[\sum_{m=1}^{\infty} \sin \left(\frac{m \pi x}{l}\right) \dot{H}_{m}^{I}\left(s_{i}, T\right)\right]^{n} d s_{i}+\int_{T}^{t}\left[\sum_{m=1}^{\infty} \sin \left(\frac{m \pi x}{l}\right) \dot{H}_{m}^{I I}\left(s_{i}, T\right)\right]^{n} d s_{i}\right]$
$\ddot{\eta}_{n}(x, t)=\lambda E\left[P_{i}^{n}\right]\left[\int_{0}^{T}\left[\sum_{m=1}^{\infty} \sin \left(\frac{m \pi x}{l}\right) \ddot{H}_{m}^{I}\left(s_{i}, T\right)\right]^{n} d s_{i}+\int_{T}^{t}\left[\sum_{m=1}^{\infty} \sin \left(\frac{m \pi x}{l}\right) \ddot{H}_{m}^{I I}\left(s_{i}, T\right)\right]^{n} d s_{i}\right]$

where $s_{i}=t-t_{i}$ and $E[$.$] denotes expectation. All semi-invariants are analytical but the$ corresponding formulae become lengthy and are not given in this paper. From the semiinvariants the central moments of the responses can be obtained as well as the coefficient of skewness $\gamma_{1}(x, t)=\eta_{3}(x, t) / \eta_{2}^{3 / 2}(x, t)$ related to the asymmetry of the density of the distribution and the coefficient of kurtosis $\gamma_{2}(x, t)=\eta_{4}(x, t) / \eta_{2}^{2}(x, t)+3$ related to the flattening of the density of the distribution near its center. Using these expressions a measure of the bandwidth of the response may be obtained as

$\epsilon(x, t)=\frac{\sigma_{\dot{v}}^{2}(x, t)}{\sigma_{v}(x, t) \sigma_{\ddot{v}}(x, t)}$

where $\sigma_{\dot{v}}(x, t)$ and $\sigma_{\ddot{v}}(x, t)$ are the standard deviations of the first- and second order derivative processes of the response. The limiting values of $\epsilon(x, t)=0$, or 1 mean that the response is ideally wide band or ideally narrow band, respectively. The moments of the response and its derivatives can be used in different models to estimate the reliability measures of the beam under the specified loading conditions. Results are presented here only for the first fundamental mode with $\mathrm{m}=1$. The models sought must utilize higher order moments to account for the non-Gaussian behaviour of the response. In Winterstein's model [10] the marginal distribution of the response $v(x, t)$ can be matched by applying an appropriate functional transformation $g(U(x, t))$ for a Gaussian process $U(x, t)$. The Gaussian response process $U(x, t)$ has the value $u(x, t)$ when the actual non-Gaussian response process $V(x, t)=g(U(x, t))$ is equal to $v(x, t)$. Dropping the arguments $x$ and $t$, the mean excursion rate is approximated as $[10]$

$\vartheta^{+}(v)=\vartheta_{0}^{+} \exp \left(-\frac{u^{2}(v)}{2}\right)$

where $\vartheta_{0}^{+}$is the zero up crossing rate written as

$\vartheta_{0}^{+}=\frac{1}{2 \pi} \frac{\sigma_{\dot{v}}}{\sigma_{v}}$

and $u(v)$ is given by $\left.\left.u(v)=\left(v_{0} / \kappa\right)-c_{3}\left(v_{0} / \kappa\right)^{2}-1\right)-c_{4}\left(v_{0} / \kappa\right)^{3}-3\left(v_{0} / \kappa\right)\right)$ for $\gamma_{2}<3$ and $u(v)=\left[\sqrt{\zeta^{2}(v)+k}+\zeta(v)\right]^{1 / 3}-\left[\sqrt{\zeta^{2}(v)+k}-\zeta(v)\right]^{1 / 3}$ for $\gamma_{2} \geq 3, v_{0}=\left(v-\mu_{v}\right) / \sigma_{v}$ and $\varsigma(v)=1.5 \beta\left[e+\left(v_{0} / \kappa\right)\right]-e^{3}$. The constants $h_{3}, h_{4}, c_{3}, c_{4}, \kappa, e, \beta$, and $k$ are defined in [10]. Similarly, a four-moment Gram-Charlier series expansion of the density function of the response can be defined. 
For a narrow band stress response $S(x, t)$ with a stress range $R$, the increase in fatigue damage $D(t)$ is written according to Miner's rule as

$\Delta D=C R^{b}$

where $C$ and $b$ are material constants. In the case of a non-Gaussian stress response $S(x, t)$, a monotonic function $g$ can be used to relate $S(x, t)$ to a standard Gaussian process $U(x, t)$ as

$S(x, t)=g(U(x, t))$

Winterstein then showed that the mean damage rate is $[10]$

$\chi_{D}=\frac{E[D(t)]}{t}=C \frac{1}{2 \pi} \frac{\sigma_{\dot{S}}}{\sigma_{S}}\left(2 \sqrt{2} \sigma_{S}\right)^{b}\left(\frac{b}{2}\right) !\left[1+b(b-1) h_{4}\right]$

where $\sigma_{S}$ and $\sigma_{\dot{S}}$ are the standard deviations of the stress response process and its velocity process. The first term in Eq. (20) is the damage rate of a narrow band Gaussian stress response and the second term is the correction factor for the non-Gaussian behavior of the response. The mean fatigue life of the beam then is inversely proportional to $\chi_{D}$. If necessary adjustments for broad band processes can be made.

\section{Some Numerical Results and Discussion}

Three types of motion are considered: the load may start from a low speed $c_{0}$ and uniformly accelerate to a certain speed $c$, it may start from a specific speed $c$ and uniformly decelerate to a low speed $c_{0}$, or it may move with a constant speed $c$. The speed $c$ is defined in terms of a dimensionless speed parameter $\alpha$ which is defined as $\alpha=c / c_{c r}$ where $c_{c r}$ is the critical speed $c_{c r}=\left(\omega_{1} l\right) / \pi$ as defined in [11]. Results are obtained using the following data: $\lambda=2[1 / \mathrm{sec}], l=30[\mathrm{~m}], P=60[\mathrm{kN}], M=2600[\mathrm{~kg} / \mathrm{m}], E=2 \times 10^{8}$ $\left[\mathrm{kN} / \mathrm{m}^{2}\right], \xi=0.01$, or $0.05, \omega=25[\mathrm{rad} / \mathrm{sec}], b=4$, and $C=7.8 \times 10^{6}\left[\mathrm{kN} / \mathrm{m}^{2}\right]$. Stresses are determined at the mid-span of a beam with height equal to $2 \mathrm{~m}$. Figure 2 (i-iv) shows the response moments for damping coefficient $\xi=0.01$ reproduced from [7]. Figure 3 (i-v) shows the bandwidth measure and excursion rates versus the speed parameter $\alpha$ for the same damping coefficient and the three acceleration regimes. As anticipated, the response process approaches a narrow band process as $\alpha$ increases (Figure 3(i)). The zero excursion rate is shown in Figure 3 (ii). Figure 3 (iii-v) shows the Gaussian, Gram-Charlier, and Winterstein excursion rates. The level of excursion is taken as $v_{l}=3 \sigma_{v}+\mu_{v}$. The Gaussian excursion rate is unconservative in comparison with the Charlier and Winterstein excursion rate models. The last two models yield almost the same excursion rate for this level of excursion. As the level of excursion increases the differences among the three models are more apparent than the case of lower level of excursion because the tail regions of the distribution of the response become more crucial and Winterstein's model is found to be more conservative than the other two models. Figure 4 (i-iv) shows the fatigue life versus $\alpha$ for the beam under consideration. In here results are presented for the Gaussian and Winterstein models only. Two values of damping coefficients are used. Variations in the fatigue life of the beam due to the use of different models is not as important as in the case of extreme value calculations. 

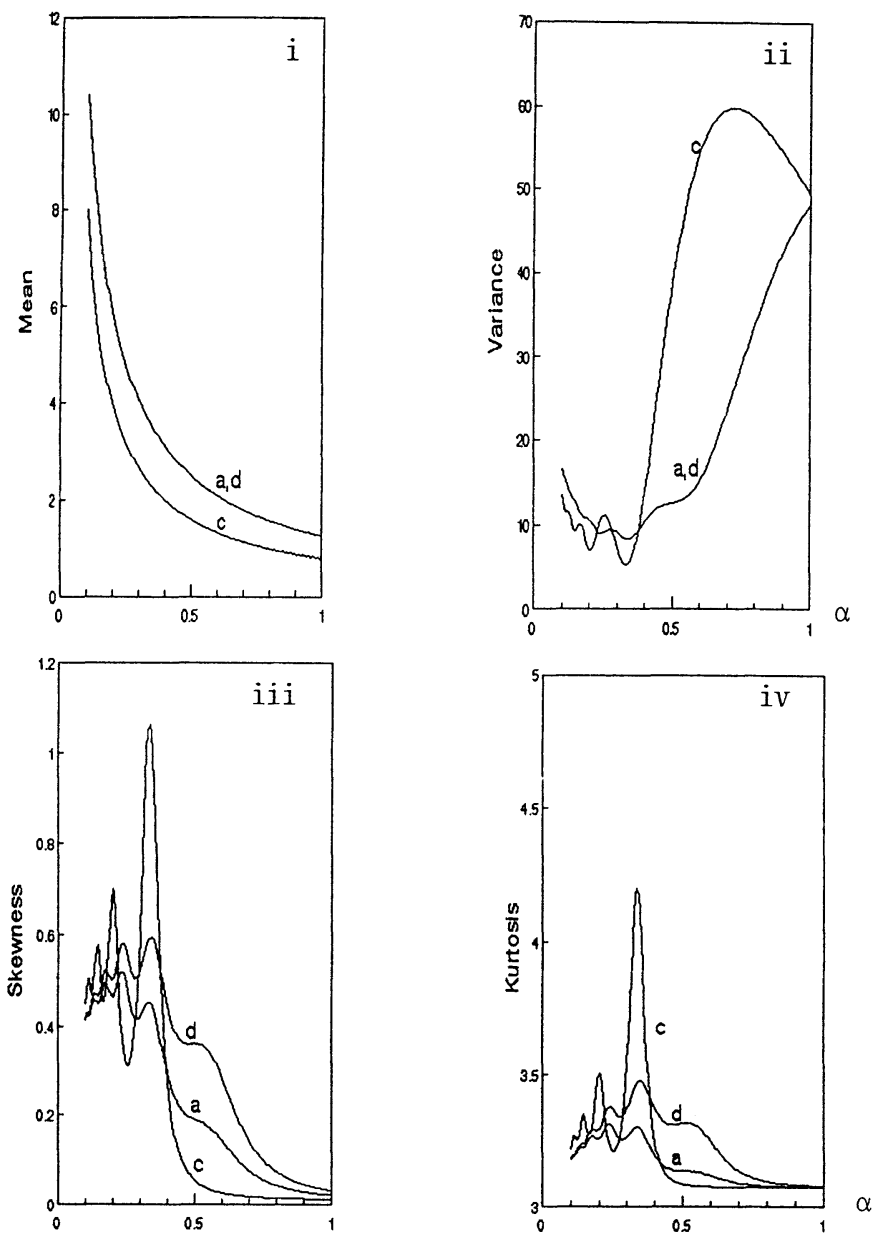

Figure 2. i) Mean value ii) Variance iii) Skewness iv) Kurtosis of the response at midspan of the beam versus the speed parameter

\section{References}

1. C.C. Tung (1967) Proc. ASCE, J. Eng. Mech. Div. 93, 73-94. Random response of highway bridges to vehicle loads.

2. C.C. Tung (1969) Proc. ASCE, J. Eng. Mech. Div. 95, 41-57. Response of highway bridges to renewal traffic loads.

3. C.C. Tung (1969) Proc. ASCE, J. Eng. Mech. Div. 95, 1417-1428. Life expectancy of highway bridges to vehicle loads. 

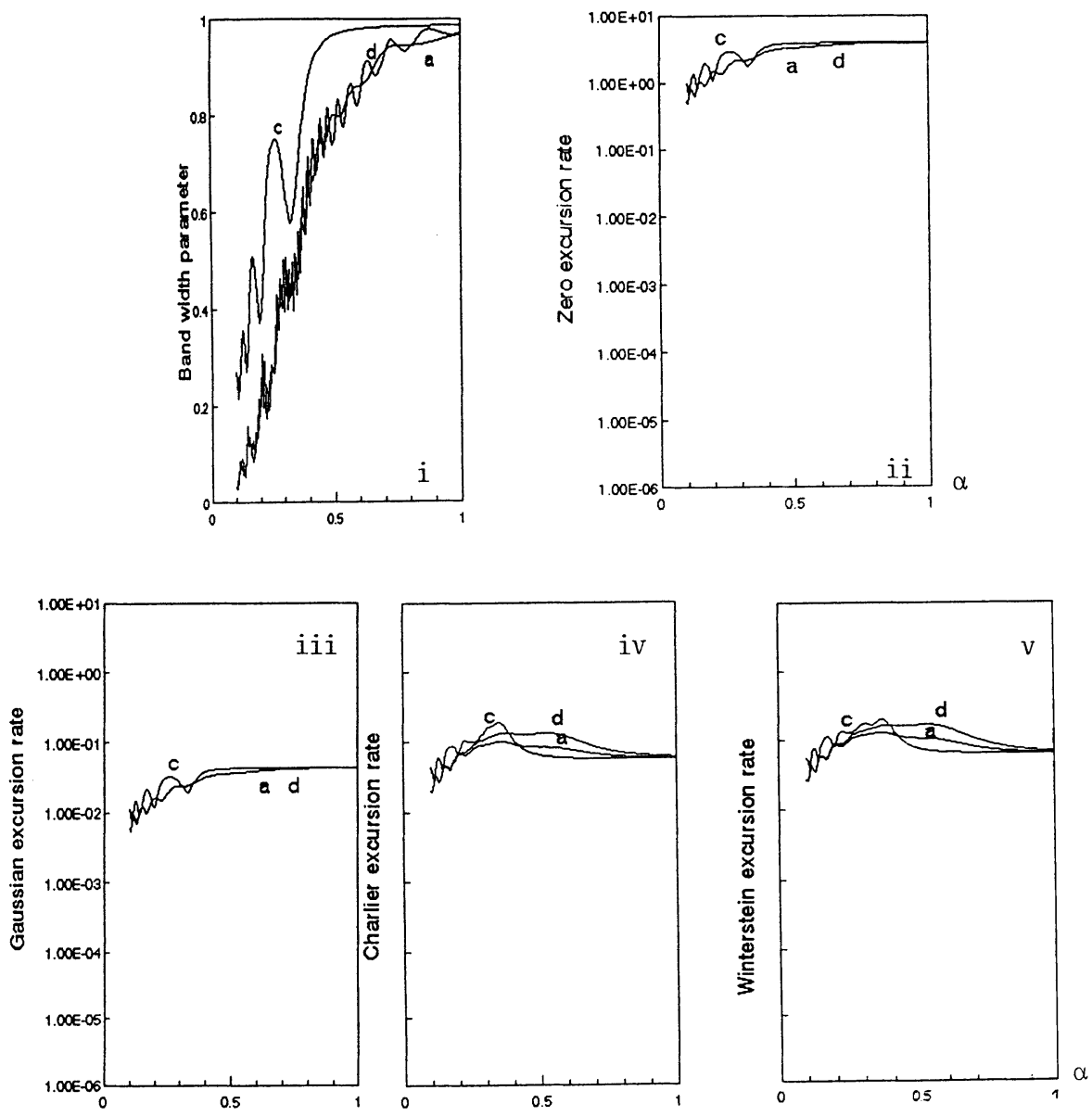

Figure 3. i) Band width parameter ii) Zero excursion rate iii-v) Excursion rates; $v_{l}=3 \sigma_{v}+\mu_{v}$ a: acceleration $\mathrm{d}$ : deceleration $\mathrm{c}$ : constant velocity; $\lambda=2.0 / \mathrm{sec} ; \xi=0.01$

4. R. Sieniawska and P. Sniady (1990) Journal of Sound and Vibration 136, 177-185. First passage problem of the beam under a random stream of moving forces.

5. R. Sieniawska and P. Sniady (1990) Journal of Sound and Vibration 140, 31-38. Life expectancy of highway bridges due to traffic load.

6. L. Fryba (1980) Journal of Sound and Vibration 70, 527-541. Estimation of fatigue life of railway bridges under traffic loads.

7. H.S. Zibdeh and R. Rackwitz (1994) Proceedings of the second international conference on computational stochastic mechanics, Athens. Random stream of moving loads on an elastic beam. 

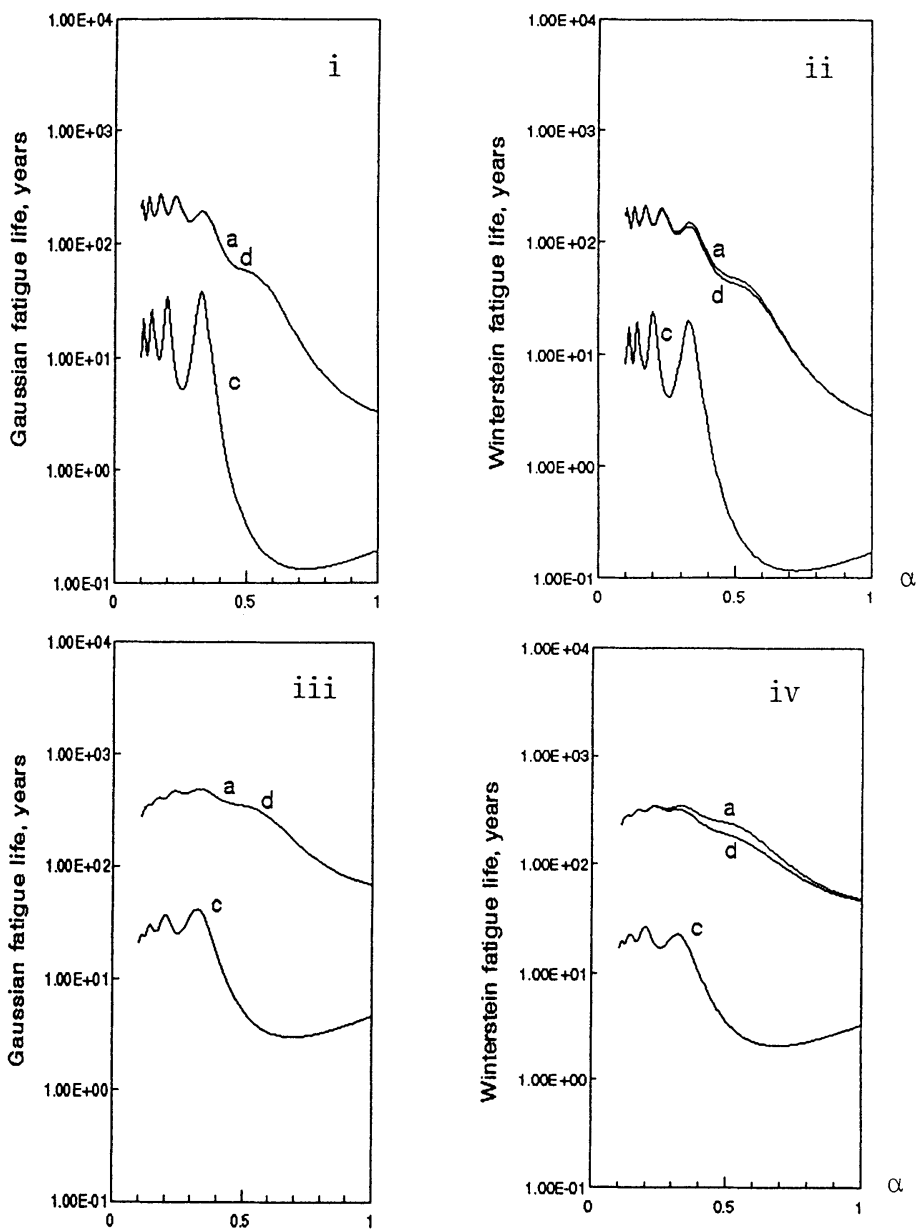

Figure 4. i, ii) Fatigue lives $\xi=0.01$ iii, iv) Fatigue lives $\xi=0.05$; a: acceleration d: deceleration c: constant velocity; $\lambda=2.0 / \mathrm{sec}$

8. H.S. Zibdeh (1994) Submitted for Publication. Stochastic vibration of an elastic beam due to random moving loads and deterministic axial forces.

9. J.B. Roberts (1966) Journal of Sound and Vibration 4, 51-61. On the response of a simple oscillator to random impulses.

10. S.R. Winterstein (1988) ASCE J. Eng. Mech. Div. 114, 1772-1790. Nonlinear vibration models for extremes and fatigue.

11. L. Fryba (1972) Vibration of solids and structures under moving loads, Noordhoff International Publishing. 